\title{
Retrorectal Tumors
}

\author{
Jeffrey A. Neale, M.D. ${ }^{1,2}$
}

\begin{abstract}
Tumors that arise in the retrorectal (presacral) space are uncommon lesions that present with nonspecific signs and symptoms, which lead to difficult diagnoses. For complete evaluation of the lesion, cross-sectional imaging is required to determine the extent of resection and the appropriate surgical approach. Surgical removal leads to favorable outcomes for patients with benign purely cystic retrorectal tumors. Preoperative tissue diagnosis with transperineal and transsacral biopsies of solid or heterogeneous cystic lesions, are essential to determine the necessity of neoadjuvant therapy, which may decrease local recurrence after surgery and avoid an unnecessary delay in systemic therapy.
\end{abstract}

KEYWORDS: Retrorectal tumors, presacral, treatment, prognosis, outcomes

Objectives: After completing the article, the reader will be able to: (1) describe the anatomy of the presacral space; (2) summarize the types of retrorectal tumors; and (3) provide an overview of the diagnosis, treatment, and outcome of retrorectal tumors.

The retrorectal (presacral) space is an area that can lodge a group of heterogeneous and rare tumors. These lesions range from benign cysts to complex malignant masses that can invade the surrounding pelvic structures. These tumors are often indolent and produce nonspecific signs and symptoms. Because of delayed diagnosis, the tumors can reach a substantial size and may involve other organs at the time of their diagnosis. In recent years, the advances in imaging modalities, understanding of tumor biology and adjuvant therapy, have improved the management of these tumors.

The incidence of retrorectal tumors in the general population is unknown. However, several retrospective series found that between one and six patients are diagnosed yearly in tertiary referral centers. ${ }^{1-3}$ Uhlig and Johnson ${ }^{4}$ demonstrated the incidence of two presacral tumors per year in a metropolitan population at a nontertiary referral center and Jao et $\mathrm{al}^{5}$ found that retrorectal tumors accounted for 1 in 40,000 hospital admissions.

Although infrequent, an understanding of the anatomy, presentation, ideal imaging modalities, and management of these lesions, will help the attending physician to avoid the significant morbidity and complications associated with incorrect diagnosis and inappropriate management. ${ }^{1}$

\section{CLINICAL ANATOMY AND PHYSIOLOGY}

The retrorectal space lies above the presacral fascia, between the upper two-thirds of the rectum and sacrum. This site contains totipotential cells, and is where fusion of the embryologic hindgut and the neuroectoderm occurs. A variety of tumors found in this space are extensions of tumors arising from adjacent tissues ${ }^{6-8}$ The presacral space contains connective tissue, the superior rectal vessels, middle sacral artery, and components

\footnotetext{
${ }^{1}$ Department of Colon and Rectal Surgery, Henry Ford Hospital, Detroit, Michigan; ${ }^{2}$ The Colorectal Institute, Fort Myers, Florida. Address for correspondence and reprint requests: Jeffrey A. Neale, M.D., The Colorectal Institute, 2675 Winkler Ave., Ste. 130, Fort Myers FL 33901 (e-mail: JeffNeale@hotmail.com).

Uncommon Colorectal Neoplasms; Guest Editor, Craig A.
}

\section{Reickert, M.D.}

Clin Colon Rectal Surg 2011;24:149-160. Copyright (C) 2011 by Thieme Medical Publishers, Inc., 333 Seventh Avenue, New York, NY 10001, USA. Tel: +1(212) 584-4662.

DOI: http://dx.doi.org/10.1055/s-0031-1285999.

ISSN 1531-0043. 
of both sympathetic and parasympathetic nervous systems (Fig. 1). Injury to these vascular and neural structures may have profound effects on rectoanal physiology and cause substantial neurologic and musculoskeletal morbidity. Anorectal function can be maintained if all unilateral nerve roots are sacrificed, or if the upper three sacral nerve roots are left intact bilaterally. However, if the S3 nerve roots are sacrificed bilaterally, the external sphincter will not contract during dilation of the rectum, thus causing various degrees of incontinence. ${ }^{9}$ If greater than half of the vertebral body of S1 remains intact after sacrectomy, pelvic stability will be maintained. However, if this area underwent radiation preoperatively, spinopelvic stability may require augmentation with fusion. ${ }^{5,8}$

\section{Classification}

A variety of classifications systems have been proposed to categorize the diverse nature of presacral tumors. Uhlig and Johnson ${ }^{4}$ first classified retrorectal tumors into broad categories: congenital, neurogenic, osseous, inflammatory, and miscellaneous. Dozois et $\mathrm{al}^{8}$ modified Uhlig and Johnson's classification and subcategorized them into malignant and benign entities - the therapeutic approach is different for each (Table 1).

\section{CONGENITAL LESIONS}

Congenital lesions are thought to arise from the remnants of embryonic tissue and can present as either cystic or solid lesions. Cystic congenital lesions include

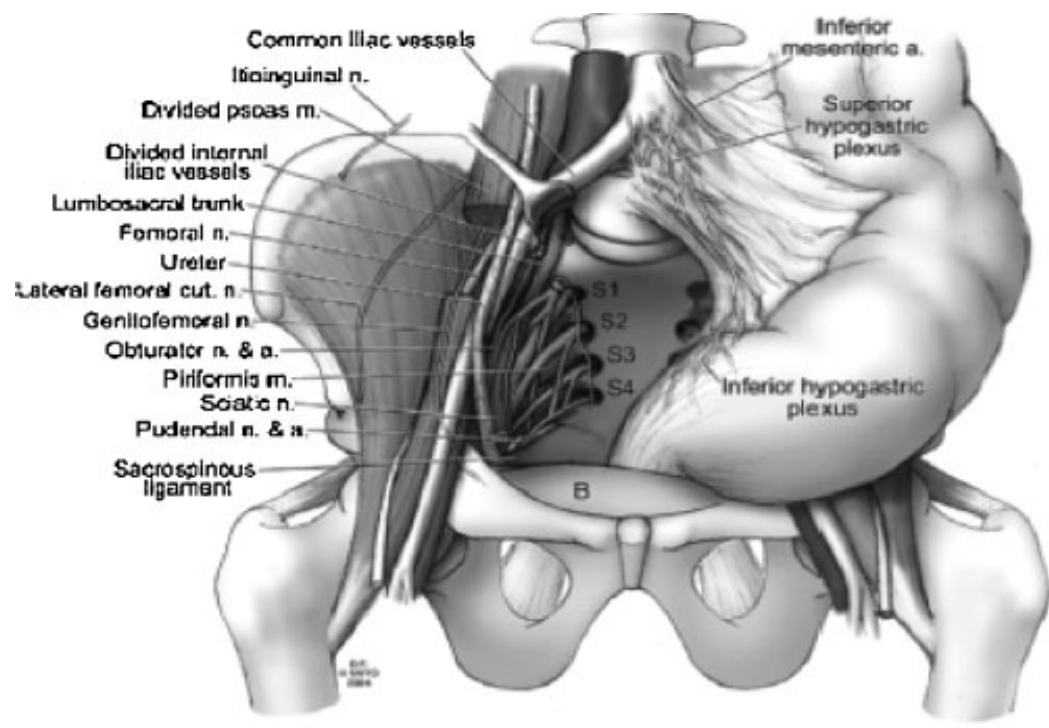

A

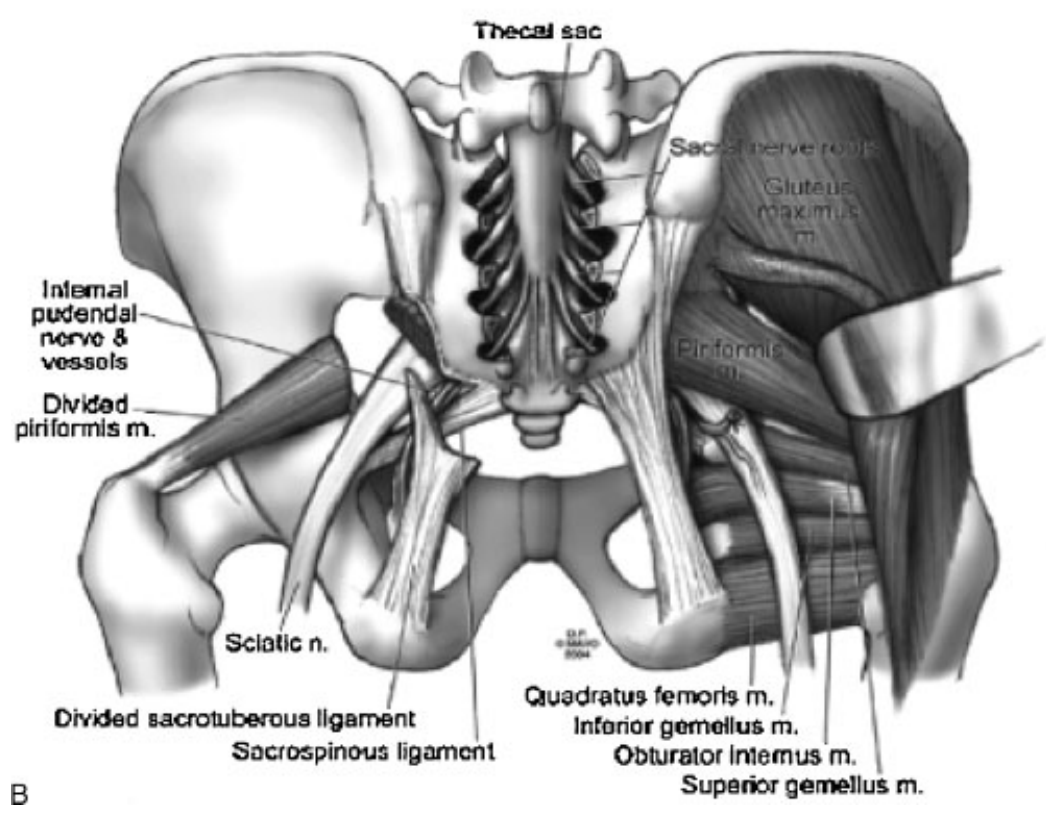

Figure 1 (A) Anterior and (B) posterior view of pelvic anatomy. From Dozois et al. ${ }^{8}$ Reprinted with permission of the American Society of Colon and Rectal Surgery. 


\section{Table 1 Classification of Presacral Tumors}

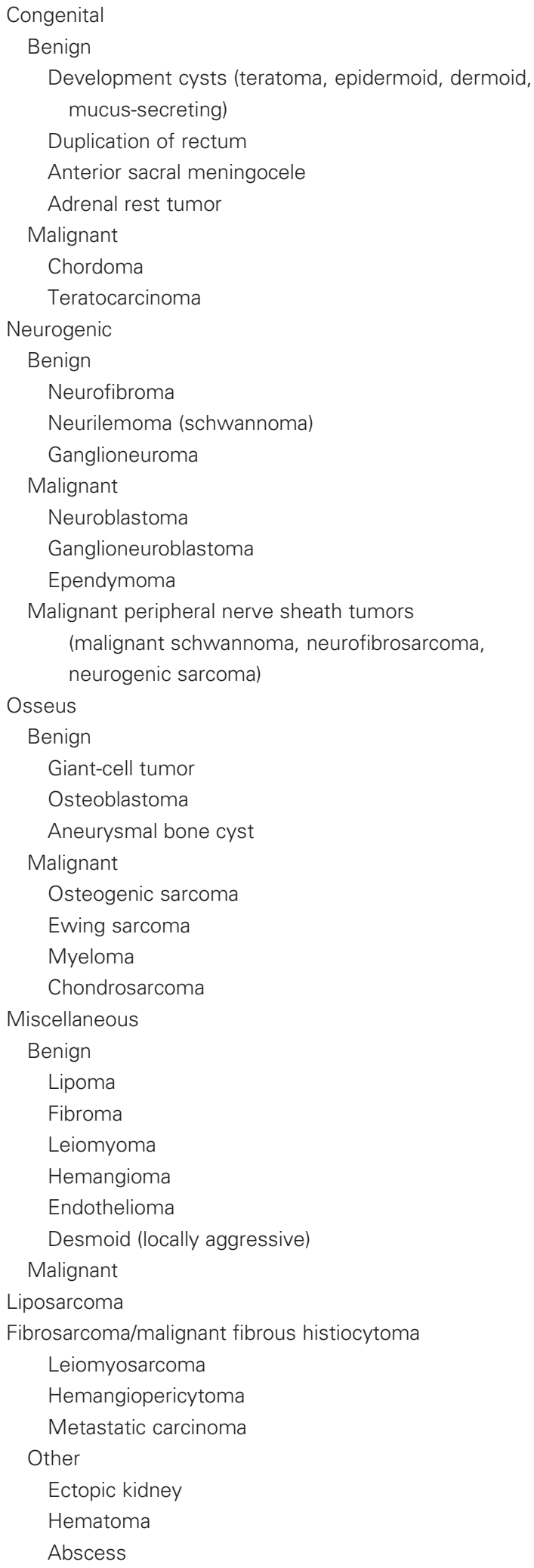

From Dozois et al. $^{8}$ Reprinted with permission of the American Society of Colon and Rectal Surgery. developmental cysts and anterior meningoceles. The solid congenital lesions consist of teratomas, sacrococcygeal chordomas, and adrenal rest tumors. Congenital lesions are the most common retrorectal tumors, accounting for nearly two-thirds of all tumors in the presacral space. They are usually benign and are more common in females. ${ }^{4,5,10}$

\section{CYSTIC LESIONS}

\section{Developmental Cysts}

Developmental cysts are the most common congenital lesions; they may arise from all three embryonic germ layers and are more common in women. ${ }^{1}$ Developmental cysts can be further classified based on cell layer of origin and are divided into epidermoid, dermoid, duplication (enterogenous), and tail gut cysts.

\section{EPIDERMOID CYSTS AND DERMOID CYSTS}

Epidermoid and dermoid cysts are the direct result of closure failure of the ectodermal tube. Epidermoid cysts are composed of stratified squamous cells; they are typically benign unilocular lesions that do not contain skin appendages. Unlike epidermoid cysts, dermoid cysts have stratified squamous epithelium with skin appendages (sweat glands, hair follicles, sebaceous cysts). Both of the type of cysts may communicate with the skin and be associated with a postanal dimple or sinus. When misdiagnosed and managed as a perirectal abscesses, fistula in ano, or pilonidal disease, there is a secondary infection rate of $\sim 30 \%{ }^{5,10,11}$

\section{DUPLICATION CYSTS (ENTEROGENOUS)}

Duplication cysts occur secondary to sequestration of embryogenesis of the developing hindgut. ${ }^{12}$ Because they arise from endodermal tissue, they can be lined with squamous, cuboidal, columnar, or transitional epithelium. These tumors usually have a multilobular appearance with multiple satellite lesions and one dominant lesion. ${ }^{8}$ Similar to epidermoid and dermoid cysts, they are more common in women and may become infected. Although generally benign, cases of malignant degeneration have been reported. ${ }^{6}$

\section{TAIL GUT CYSTS (CYSTIC HARMATOMAS)}

Tail gut cysts are multinodular, uncapsulated, and usually well-circumscribed cysts. They arise from a portion of the embryonic tail, which fails to regress. ${ }^{13}$ These lesions resemble the intestinal tract with components of squamous, columnar, or transitional epithelium. Tail gut cysts can be differentiated from epidermoid, dermoid, and duplication cysts histologically. Epidermoid and dermoid cysts lack the presence of glandular or transitional epithelium; duplication 
cysts have a defined muscular wall with a myenteric plexuses. In general, these are benign lesions and although rare, malignant transformation has been reported. ${ }^{4}$

\section{Anterior Sacral Meningocele}

Anterior meningocele and myelomeningocele develop secondary to herniation of the dural sac through a defect in the anterior sacrum. This unilateral defect results in a sacrum that demonstrates a rounded concave border without bony destruction on plain radiograph. This is pathognomonic, and called the "scimitar sign" (Fig. 2). This defect can occur in combination with presacral cysts or lipomeas and is associated with congenital abnormalities, which include urinary tract or anal malformations, uterine or vaginal duplication, spina bifida, and a tethered spinal cord. ${ }^{8}$ A typical presenting symptom is a headache associated with defecation. This can be attributed to the compression-induced increase in cerebrospinal fluid (CSF) pressure due to the continuity between the dural sac and subdural space. ${ }^{1,5}$ Patients may also present with symptoms secondary to mass effect and include constipation, urinary symptoms, and lower back pain. ${ }^{10,14}$ Biopsy or aspiration are prohibited as this may result in life-threatening meningitis. ${ }^{6,15}$ Surgical management requires ligation of the dural defect. ${ }^{8}$

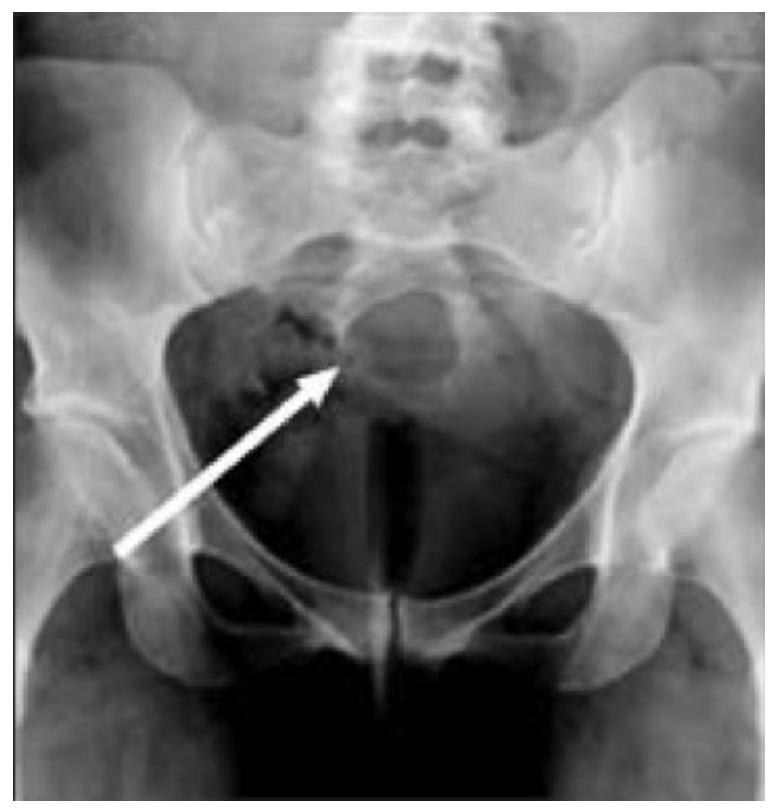

Figure 2 "Scimitar sign": Pathognomonic for anterior meningocele. Reprinted from Bou-Assaly W, Iner A, Delaney L, Gunderman R. AJR teaching file: child with chronic constipation. Am J Roentgenol 2007;189(3 Suppl): S29-S31, with permission of the American Roentgen Ray Society.

\section{SOLID LESIONS}

\section{Teratomas}

Teratomas are true neoplasms. They include all three germ layers; therefore, they include epithelium of the gastrointestinal tract, respiratory tract, and nervous system. These lesions can be solid or cystic and often contain both components. If germ cell components are present, teratomas have the potential to undergo malignant degeneration to squamous cell carcinoma or rhabdomyosarcoma; up to $10 \%$ will undergo degeneration if left untreated. ${ }^{1}$ Teratomas are more common in female compared with male patients and are more frequently found in the pediatric population. In children, teratomas are associated with anomalies of the anorectum, urinary tract, and vertebrae. ${ }^{16}$ In the adult population there is a risk of 40 to $50 \%$ malignant degeneration and which increases with incomplete resection. ${ }^{17,18}$ These lesions tend to adhere to the coccyx and surgical approach requires en bloc coccygectomy. ${ }^{1}$

\section{Sacrococcygeal Chordomas}

Chordomas arise from the notochord, which extends from the base of the occiput to the caudal limit of the embryo. Fuchs et al from the Mayo clinic reported 52 cases over 21 years, 2.5 cases per year. ${ }^{19}$ They are the most common malignancy in the retrorectal space and may occur anywhere along the embryologic notochord with 30 to $50 \%$ occurring in the sacrococcygeal region. ${ }^{20}$ They have a predisposition to males and presentation before the third decade of life is rare. These tumors are slow-growing invasive cancers and demonstrate bony destruction on imaging. They have a tendency to invade adjacent structures and metastasize to lung, liver, and bone in $20 \%$ of cases. ${ }^{9,21}$ These tumors often reach substantial size because of delayed diagnosis. ${ }^{8}$ Patients may be asymptomatic, present with vague complaints including positional buttock, pelvic, or lower back pain; or they may present with specific symptoms secondary to invasion, including impotence and incontinence. ${ }^{5}$ Local recurrence rates are high despite radical resection; the 10 -year survival rate is only 9 to $35 \% .^{10,22,23}$

\section{Adrenal Rest Tumor}

These are extremely rare tumors and are treated as ectopic adrenal tumors (pheochromocytoma). 1,13

\section{NEUROGENIC TUMORS}

Neurogenic tumors represent $\sim 10 \%$ of retrorectal tumors and are the second most common presacral lesion after congenital lesions. They typically arise from peripheral nerves and $\sim 85 \%$ are benign. ${ }^{6,12}$ The benign lesions consist of neurofibromas, neurilemomas 
(schwannoma), and ganglioneuromas. The malignant neurogenic lesions include neuroblastoma, ganglioneuroblastoma, ependymoma, and malignant peripheral nerve sheath tumors (malignant schwannoma, neurofibrosarcoma, and neurogenic sarcoma). ${ }^{8}$ Neurogenic tumors are slow-growing tumors and may cause nonspecific symptoms; hence, they may be of considerable size when diagnosed. If symptoms are present, pain distribution and neurologic dysfunction are related to the route of the affected nerve.

\section{OSSEOUS TUMORS}

Osseous tumors also represent $10 \%$ of retrorectal tumors. They can arise from bone, cartilage, fibrous tissue, and marrow. The benign presacral tumors include giant-cell tumor, osteoblastoma, and aneurysmal bone cyst. Malignant osseous lesions include osteogenic sarcoma, Ewing sarcoma, myeloma, and chondrosarcoma. Because of rapid growth, these tumors achieve a considerable size. If malignant, they are typically advanced and demonstrate osseous destruction or calcification of soft tissue on plain radiograph. Malignant osseous lesions have frequent lung metastases and have an overall poor prognosis. ${ }^{1,6,8,10}$

\section{MISCELLANEOUS TUMORS}

Miscellaneous tumors account for 10 to $25 \%$ of all retrorectal tumors and include lipoma, fibroma, leiomyoma, hemangioma, endothelioma, desmoid (locally aggressive), liposarcoma, fibrosarcoma, malignant histiocytoma, leiomyosarcomas, hemangiopericytoma, metastatic adenocarcinoma, and inflammatory tumors. ${ }^{8,12}$ Inflammatory tumors should be considered secondary reactions to foreign substances such as barium and suture material, or represent an extension of infection from either the perirectal space or abdomen, i.e., pelvic sepsis, Crohn disease, and perforated diverticulitis. $^{21,24,25}$

\section{DIAGNOSIS AND MANAGEMENT}

\section{History and Physical Examination}

The nature of retrorectal tumors is that of an indolent course. They are usually found incidentally while performing a pelvic or rectal examination. Most symptoms can be attributed to compression or invasion of pelvic viscera or nerves. ${ }^{1,7}$ Patients who present with symptoms may have a spectrum of complaints. They may be vague and complain of longstanding lower back pain, which may worsen with sitting and ameliorate with walking and standing. Patients who present with specific complaints of constipation, rectal and urinary incontinence, and sexual dysfunction will have a large tumor or one that has invaded the sacral root. ${ }^{1}$ Patients may also complain of perineal discharge and they may have midline dimpling just posterior to the anus or the gluteal muscle. This may lead to misdiagnosis of a fistula or pilonidal disease. ${ }^{1,26}$ In general, pain is a presenting symptom for malignant retrorectal tumors and for those benign tumors that have had a secondary infection. ${ }^{1}$ Rarely, retrorectal tumors may lead to obstructive labor in a pregnant patient and predispose her to life-threatening dystocia. Therefore, these tumors, even if asymptomatic, are removed from women of childbearing age. ${ }^{27}$

A neurologic exam must be conducted to determine any neurologic deficiency and document preoperative function. A rectal examination is essential in ascertaining the proximal level of the lesion, extent of fixation, and relationship to other pelvic viscera. A digital rectal examination will demonstrate a smooth intact rectal mucosa and a posterior extrarectal mass that displaces the posterior rectal wall anteriorly. ${ }^{7} \mathrm{~A}$ rigid or flexible sigmoidoscopy can be performed to ensure there is no transmural penetration of the tumor.

Overall, presenting symptoms of retrorectal tumors are nonspecific and the literature demonstrates a wide range of variability in physical findings. ${ }^{10,25,28}$ If one maintains a high clinical acumen and is cognoscente of the range of symptoms associated with retrorectal tumors, a delay in diagnosis can be avoided. ${ }^{1}$

\section{IMAGING}

As technology has evolved, radiographic imaging has become more sophisticated and plain radiographs have been replaced by computed tomography $(\mathrm{CT})$ scans and magnetic resonance imaging (MRI), as the imaging modalities of choice in diagnosing retrorectal tumors. Plain $\mathrm{x}$-rays also elucidate bony destruction and/or calcification of soft tissue secondary to chordomas, sarcomas, and locally aggressive tumors such as giant cell tumor, aneurysmal bone cyst, and neurilemoma. ${ }^{6,8}$ Other imaging techniques that may assist in the diagnosis and management of retrorectal tumors include angiogram and venogram, endorectal ultrasound (ERUS), and fistulograms. An angiogram and venogram can be added to MRI (MR angiogram and venogram) to determine vascular anatomy and involvement if there is distortion secondary to mass effect by the tumor. ERUS has been used to evaluate whether the lesion is cystic or solid, and to evaluate rectal involvement. Fistulograms have been used in patients who present with a chronically draining sinus to evaluate for a possible developmental cyst. ${ }^{8}$

Currently, CT used in conjunction with MRI is the gold standard in diagnosing retrorectal tumors. CT is used to demonstrate cortical bone destruction, the nature of the lesion - solid or cystic — and if the adjacent viscera is involved. The advanced capabilities associated 
with MRI to evaluate soft tissue and determine spatial relationship to other structures is extremely important in determining level and extent of resection (local vs en bloc) and surgical approach (anterior vs posterior vs combined). MRI is also more sensitive in spinal imaging and will demonstrate cord anomalies, thecal sac compression, marrow or nerve root involvement. ${ }^{1,20}$ In recent years, Zhang et $\mathrm{al}^{29}$ have demonstrated the possible utility of carbon-11 methionine-based positron emission tomography (MET PET) in evaluating the response of sacral chordomas that have undergone experimental carbon ion radiotherapy (CIRT). In comparison to ${ }^{18} \mathrm{~F}$-flurodeoxyglucose (FDG), L-methionine (MET) plays a central role in the altered metabolism of cancer cells and uptake signifies cancer cell proliferation reflecting transmethylation rate and protein synthesis of malignant tissues. ${ }^{29}$ Unlike classic FDG-PET, MET-PET will not undergo uptake into inflammatory cells such as macrophages, neutrophils, or fibroblasts and will exclude any radiation-induced inflammation on imaging. ${ }^{29}$

\section{PREOPERATIVE BIOPSY}

Historically, the role of preoperative biopsy for retrorectal tumors has been controversial. ${ }^{5,24,30-32}$ Over the past 25 years, different biopsy approaches have been advocated for needle aspiration of pelvic lesions under CT guidance. ${ }^{33-36}$ Gupta et $\mathrm{al}^{37}$ reviewed the normal pelvic anatomy as it relates to percutaneous needle biopsy of deep pelvic lesions, and described various approaches to CT-guided pelvic biopsy, including the transsacral approach and its potential complications. Yarram et $\mathrm{al}^{38}$ reviewed 111 biopsies of pelvic masses using sonography or CT-guided biopsy over a 6-year period. Twenty-seven of these lesions were either presacral or perirectal in origin. Overall, the rate of accuracy, sensitivity, and specificity for CT-guided core needle biopsy of pelvic and presacral tumors for this study was $90 \%, 84.6 \%$, and $100 \%$, respectively. Bergh et $\mathrm{al}^{22}$ were able to diagnose preoperatively 30 presacral chordomas using fine-needle aspiration (FNA), core needle biopsy, or incisional biopsy. Syed et $\mathrm{al}^{39}$ at John Hopkins Hospital reviewed the hospital's cytopathologic files, imaging, and medical records over a 20 -year period. Ninety-one cases of sacral/presacral FNA were identified with primary malignancies representing $14 \%$ of all lesions; chordomas were the most frequently found lesions (4\%). The overall rate of accuracy, sensitivity, and specificity of FNA from this study was $96 \%$, $95 \%$, and $100 \%$, respectively. ${ }^{39}$ With the advances in imaging modalities (CT and MRI) and improved knowledge of tumor biology and neoadjuvant therapy, preoperative biopsy has become a feasible technique. As advocated by Dozois et al, ${ }^{8}$ preoperative tissue diagnosis is essential for the management of solid and heteroge- neously cystic tumors. Purely cystic lesions should rarely undergo biopsy; they are usually benign and there is a risk of secondary infection. ${ }^{8}$ When indicated, preoperative biopsy results will affect the management algorithm of the patient. For example, Ewing sarcoma, osteogenic sarcoma, neurofibrosarcomas, and desmoid tumors benefit from neoadjuvant therapy. In conjunction with directing management, a preoperative biopsy also assists in determining prognostic characteristics of the tumor. ${ }^{22}$ Dozois et $\mathrm{al}^{8}$ recommends the following general rules when performing biopsies on retrorectal tumors:

1. Prior to performing a biopsy, coagulation studies must be performed as a hematoma may contaminate the involved areas.

2. The ideal approach is transperineal or parasacral because this usually is within the field of the future surgical resection.

3. The biopsy should be performed by a radiologist experienced in the diagnosis and management of pelvic tumors.

4. The surgeon and radiologist should discuss the biopsy's approach - the needle tract must be removed en bloc with the specimen.

5. Transperitoneal, transretroperitoneal, transvaginal, and transrectal biopsies are to be avoided - the tract of the biopsy must be removed en bloc. Biopsies performed transrectally or transvaginally may also lead to infection (infected meningoceles causing meningitis or death), a more difficult complete excision, or increase the probability of postoperative complications and recurrence.

\section{ADVANCES IN NEOADJUVANT AND PREOPERATIVE THERAPY}

Neoadjuvant chemotherapy is essential to the treatment of some retrorectal tumors such as Ewing sarcoma and osteogenic sarcoma. Recent advances in chemotherapy have demonstrated that imatinib, a tyrosine kinase inhibitor, promotes progression-free survival in patients with advanced chordomas. Patients with locally recurrent and metastatic chordoma respond well to chemotherapeutic agents such as cetuximab and gefitinab. ${ }^{40-42}$ Transcatheter arterial embolization (TAE) of tumors including chordomas has been performed as an adjunct to successful resection. TAE can significantly decrease intraoperative blood loss, make the surgical field clear, possibly eliminate the need for using an anterior approach, and facilitate the maximal removal of the sacral chordoma. It is an encouraging technique for excising sacral chordomas. ${ }^{43,44}$

The role of radiotherapy is not entirely defined for the management of retrorectal tumors: Tumors such as chordoma have been considered to be "radioresistant" to 
standard-dose radiation therapy. Radiation modalities, such as fractionated irradiation with charged particle carbon ion radiotherapy (CIRT), have been utilized in trials in Japan and encouraging results have been reported with respect to local control of sacral chordomas. ${ }^{29}$ Zhang et $\mathrm{al}^{29}$ utilized MET-PET to image patients with a chordomas who had undergone CIRT. Ten of the 15 patients presented with primary sacral chordomas without distant metastases; the range of tumor size was 7 to $15 \mathrm{~cm}$. CIRT was offered as the lesions were deemed unresectable and the patients were either medically unfit or declined surgery. CIRT demonstrated a decrease in metabolic activity of the chordoma on MET-PET and local control rates were in excess of $73 \%$ at 3 years contrasting with the $\sim 35 \%$ control rate reported with conventional radiation. ${ }^{29}$ Advances such as this demonstrate the positive role that radiotherapy may eventually contribute to certain types of retrorectal tumors in the neoadjuvant setting. Potential benefits include (1) a decrease in the size of large radiosensitive tumors, thus potentially sparing vital structures that would otherwise require extirpation with wide margins; and (2) direct radiation at a smaller field, thereby decreasing patient morbidity.

\section{THE MULTIDISCIPLINARY APPROACH AND PREOPERATIVE PREPARATION}

The availability of modern neoadjuvant protocols has made the multidisciplinary approach to complex malignancies essential to their management. With the combination of preoperative imaging, biopsy (when indicated), and the continued advances in adjuvant therapy, the importance of a multidisciplinary approach to retrorectal tumors must be reemphasized as of maximum benefit to the patient. This experienced team may consist of colon and rectal, spine, vascular, plastic, orthopedic and urologic surgeons; radiologists; anesthetists; and medical and radiation oncologists. Woodbridge et $\mathrm{al}^{21}$ and Dozois et $\mathrm{al}^{8}$ have both discussed the importance of this approach, which was first described by the Mayo clinic team of surgeons in $1953.8,45$ To promote surgical success, the following should be considered preoperatively:

1. If neoadjuvant therapy is to be administered, patients should have repeat imaging for restaging and to determine the adequate margins of resection.

2. The patient's nutritional status should be assessed and supplemented with total parenteral nutrition (TPN) or enteral nutrition when indicated.

3. An inferior vena cava filter should be placed.

4. When positioning the patient, ensure that nerves and soft tissue are adequately protected from pressure injury.
5. For patients with a large tumor or who have received neoadjuvant radiation, the placement of temporary ureteral catheters should be considered.

6. The anesthesia team should be well equipped to handle massive transfusion requirements. ${ }^{12,21}$

\section{SURGICAL MANAGEMENT}

The appropriate surgical approach for retrorectal tumors is ascertained by appropriate imaging (CT and MRI), which demonstrate the location, nature, and size of the lesion as well as the involvement of adjacent viscera, sacrum, or pelvic sidewalls. ${ }^{21}$ The extent of surgery is determined by the character of the tumor: Benign retrorectal tumors require complete gross resection, whereas malignant tumors will require radical resection, including en bloc resection of adjacent organs if involved. Incomplete removal will increase local recurrence and decrease survival if the tumor is malignant. The common approaches for resection of retrorectal tumors are the anterior (transabdominal), the combined abdominoperineal, and the posterior (perineal) approaches. Generally, tumors above the level of S3 will require an anterior or combined approach; small lesions below the level of S3 may be removed utilizing the posterior approach (Fig. 3). ${ }^{8}$

\section{Anterior Approach (Transabdominal)}

The anterior approach is performed when the lowest portion of the lesion is above the level of S4. To proceed with this approach, preoperative imaging must confirm that the sacrum is not involved. Traditionally, these lesions have been approached through a laparotomy; however, advanced laparoscopic technique has demonstrated a safe and efficacious alternative. For both techniques, the mesorectum should first be dissected off the anterior portion of the lesion, then the lesion must be separated from the presacral fascia. Prior to the removal

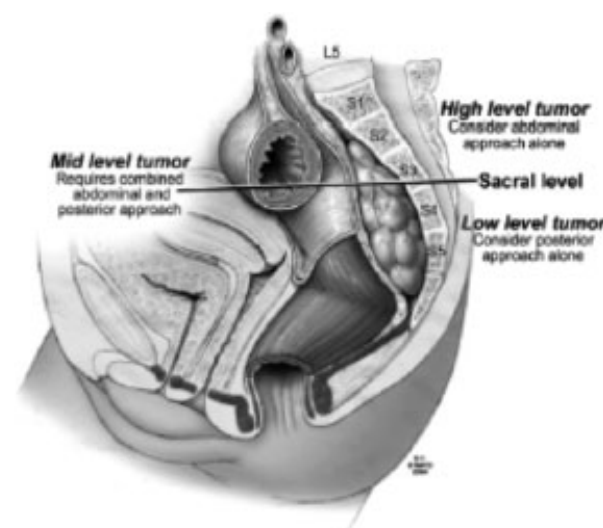

Figure 3 Proposed surgical approach based on tumor level. From Dozois et al. ${ }^{8}$ Reprinted with permission of the American Society of Colon and Rectal Surgery. 
of the tumor, the arterial supply to the lesion must be identified and ligated, as the arterial supply can be the middle sacral artery. ${ }^{7,46-48}$ To date, robotic technology has not been used in an anterior approach for presacral tumors.

\section{Combined Approach (Abdominoperineal)}

A combined approach is reserved for those lesions that are above the $\mathrm{S} 3$ vertebrae. ${ }^{5,49}$ Various techniques for positioning have been described for this approach. ${ }^{50}$ The most common positioning for this approach is to facilitate a two-team approach; this is commonly referred to as a "sloppy lateral position" (Fig. 4A). A lower midline celiotomy is performed, and the peritoneal cavity is examined for peritoneal implants or metastatic disease for malignant disease. The sigmoid colon is then mobilized, the ureters are identified and protected. The retrorectal space is then entered below the sacral promontory and the mesorectum is dissected from the presacral fascia to the upper level of the tumor. If possible, the anterior portion of tumor should then be dissected free from the mesorectum (Fig. 4B). Once complete, the posterior component of the tumor is separated from the presacral fascia. Even if the lesion is malignant, a discernable plane between the capsule of the tumor and mesorectum will allow preservation of the rectum. If this cannot be accomplished secondary to the size of the tumor or distortion of the rectum, it is obligatory to remove the rectum en bloc with the specimen, including involved sacrum or nerve roots. ${ }^{8}$ To preserve fecal and urinary continence, at least one S3 nerve root requires preservation. If it is not feasible to preserve $\mathrm{S} 3$ unilaterally, a colostomy should be created. If en bloc resection is necessary, transection of the rectosigmoid junction is performed with a stapling device at the level of the sacral promontory. The anterior rectal wall is then separated from the posterior wall of the vagina in women and seminal vesicles and prostate in men; dissection is performed to the level of the levators. If en bloc resection of the rectum is to be performed, dissection of the anus and anal canal is completed during the posterior portion of the operation. ${ }^{8}$ If the lesion is benign or considered to have a low recurrence risk, an end-to-end anastomosis can be performed with or without a protecting loop ileostomy. ${ }^{12}$ If an extended resection is to be completed or if the pelvis has undergone preoperative radiation, a well-vascularized transabdominal myocutaneous rectus (TRAM) flap (preferably the right if contemplating end colostomy) can be utilized to close the perineum. If a flap is to be used, a thick silastic mesh should be placed posterior to vital structures for protection when performing the sacrectomy. ${ }^{8}$ During the abdominal portion of the surgery, significant blood loss can occur as a result of

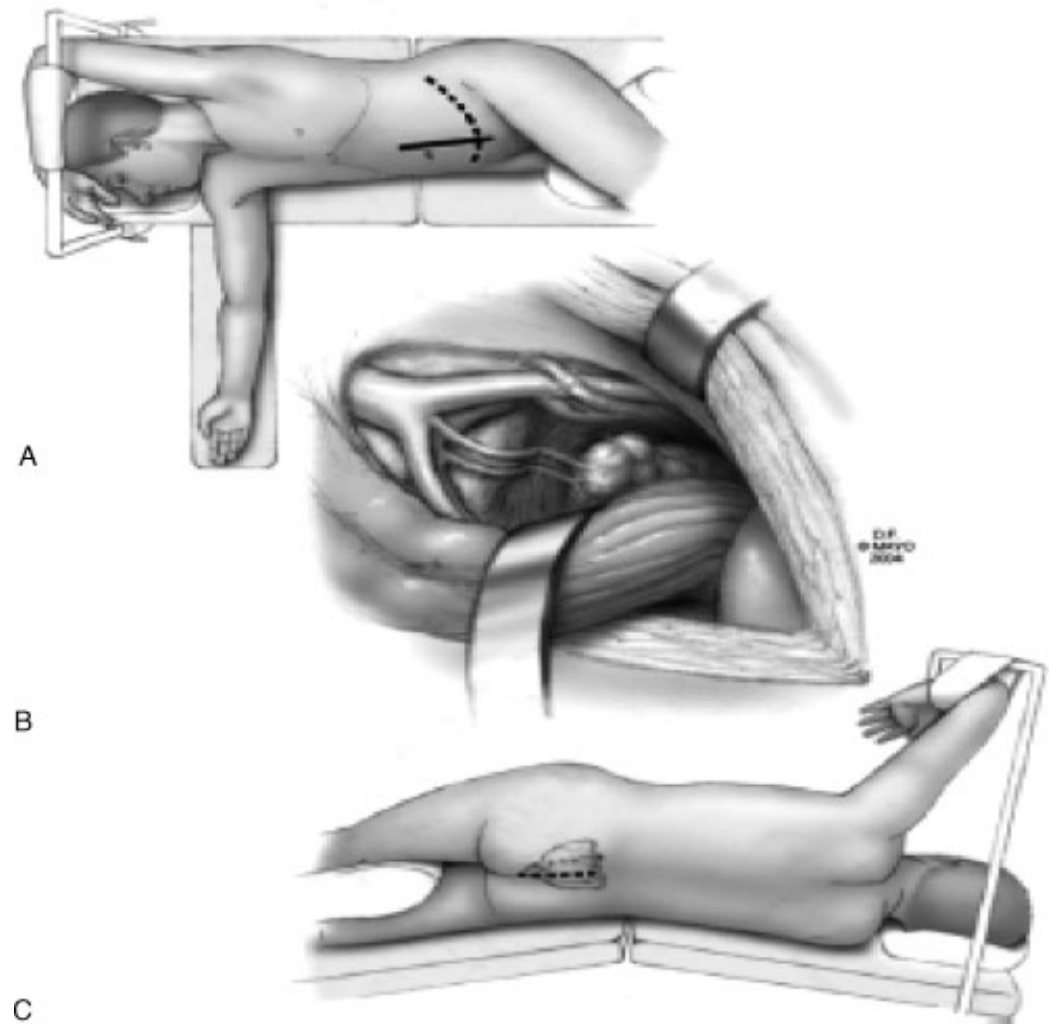

Figure 4 Combined approach (abdominoperineal). (A) Modified lateral position (sloppy lateral position), (B) exposure of pelvic structures and tumor, and (C) exposure of sacrum. From Dozois et al. ${ }^{8}$ Reprinted with permission of the American Society of Colon and Rectal Surgery. 
large complex retrosacral tumors, prior radiation, or sacrectomy. Blood loss may be minimized by ligating the middle and lateral sacral vessels and both internal iliac artery and veins. Perineal necrosis can be avoided if the inferior gluteal artery is maintained by avoiding ligation of the anterior division of the internal iliac artery. ${ }^{8}$ After closing the abdomen and maturation of the colostomy, the patient is placed in a prone position (Fig. 4C). An incision is then made over the sacrum and coccyx to the level of the anus, avoiding injury to the external sphincter. The anococcygeal ligament is then divided and the levators are retracted laterally. Dissection of the tumor from the mesorectum and possible rectal resection would have been completed during the abdominal portion of the surgery. The gluteus maximus muscles are then separated bilaterally and the sacrospinous, sacrotuberous ligaments, and piriformis muscles are transected bilaterally to expose the sciatic nerve (Fig. 5A). Posterior laminectomy may be needed to expose nerve roots and/or the thecal sac, which may require ligation and allow en bloc resection with the lower sacrum, coccyx, and involved nerve roots (Figs. 5B, C). If the sacral nerves are resected, dural tears must be recognized and repaired to avoid a cerebrospinal fluid leak or infection. If the rectum was removed en bloc with the specimen, the anus and anal canal are removed with the rectum at this time. Suction drains are placed and the perineal incision is closed in layers; if required, a myocutaneous flap is sewn into place. $^{8}$

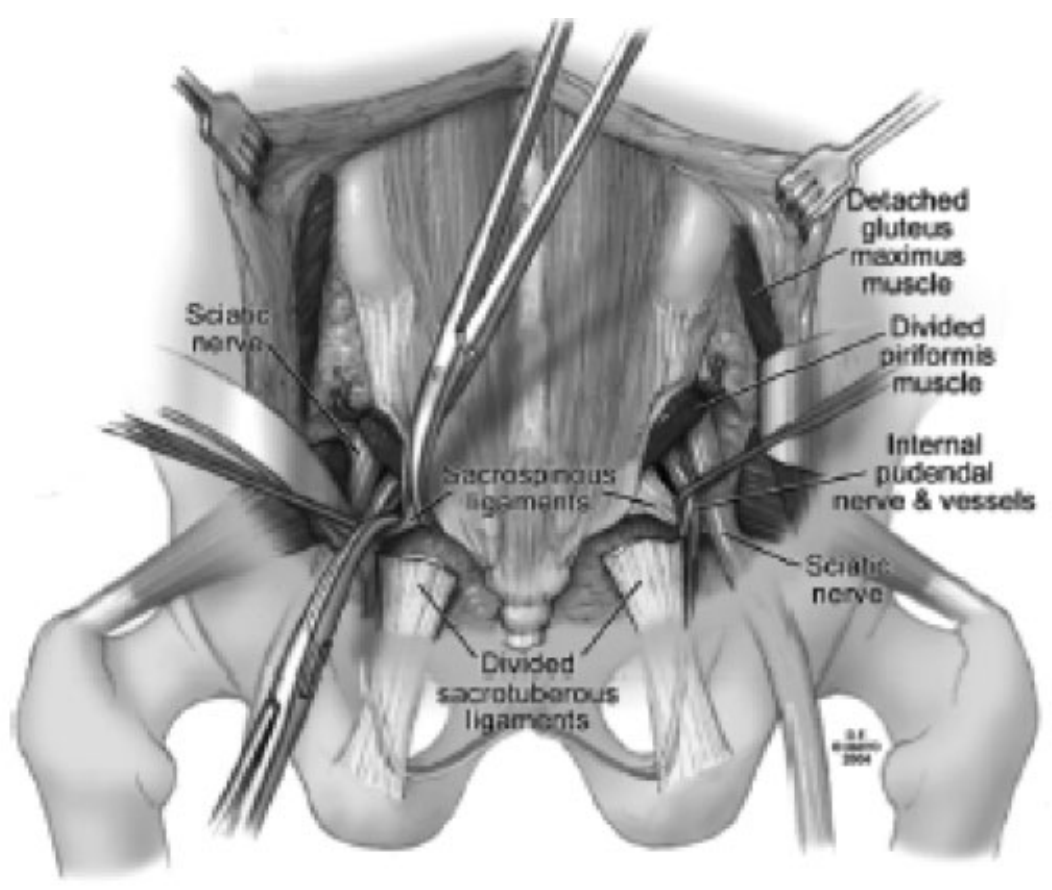

A

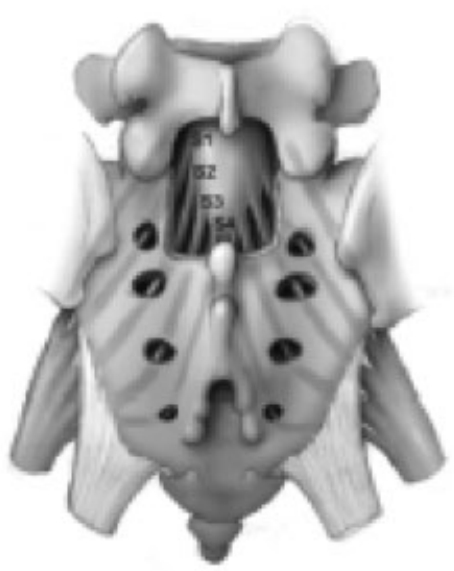

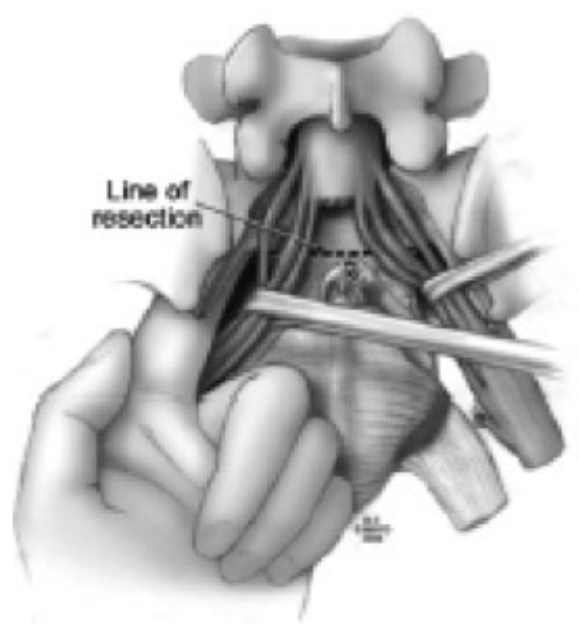

C

Figure 5 (A) Exposure of sciatic nerves, (B) exposure of sacral nerve roots, and (C) exposure of thecal sac. From Dozois et al. ${ }^{8}$ Reprinted with permission of the American Society of Colon and Rectal Surgery. 


\section{Posterior Approach (Perineal)}

The posterior approach is ideal for retrorectal tumors that do not extend above the level of S3. The patient is placed in a prone jack-knife position, the buttocks are taped apart (Fig. 6A). A midline incision is performed on the lower sacrum to the level of anoderm, ensuring preservation of the external sphincter (Fig. 6B). The anococcygeal ligament is identified and transected to expose tumor and facilitate dissection of the plane between the lesion and the mesorectum. To provide sufficient exposure for dissection the coccyx can be transected, the gluteal muscle may be detached, and sacrectomy of S4-S5 can be performed. ${ }^{49}$ Once adequate exposure is obtained, the tumor can be separated from the rectum. There is usually a identifiable fat plane between the mesorectum and retrorectal lesion if the lesion is benign. ${ }^{12}$ If lesions are very small or cystic, the surgeon may double-glove his nondominant hand, place his index finger in the anal canal and lower rectum and express the lesion into the wound by applying forward pressure with his index finger (Fig. 6C). This maneuver prevents iatrogenic injury to the rectal wall during the dissection. If the lesion is adherent to the rectum and cannot be safely dissected, a portion of the rectal wall can be excised with the lesion. The defect is then repaired in two layers. ${ }^{1,12}$

\section{OUTCOMES}

Overall survival for benign tumors is $\sim 100 \%$. Recurrence rates vary and are dependent on extent and

A
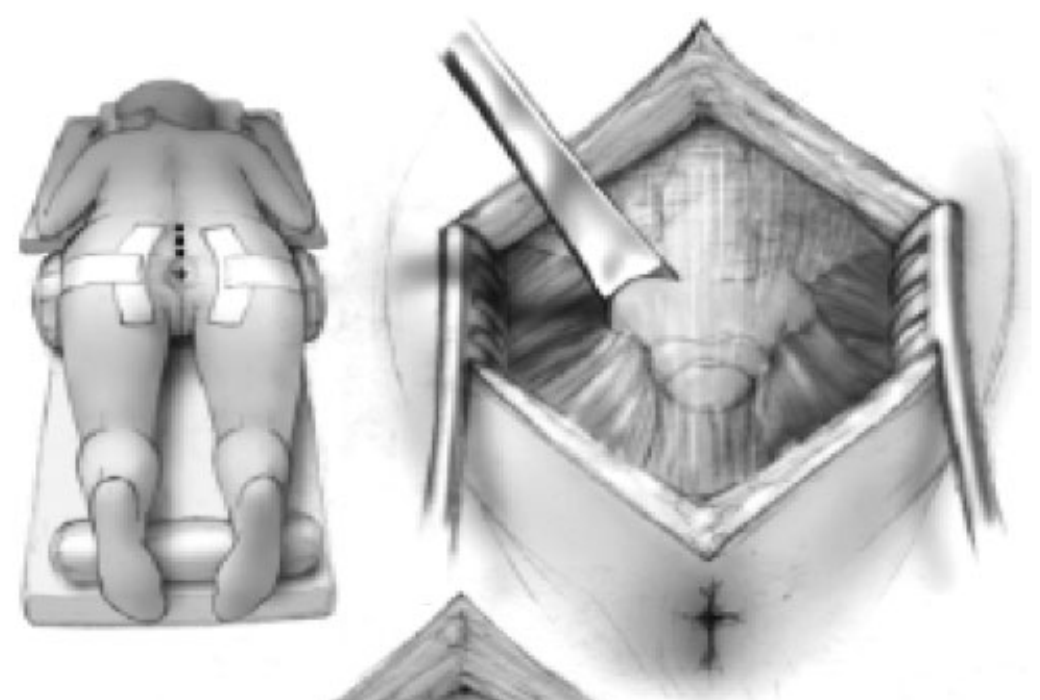

B

C

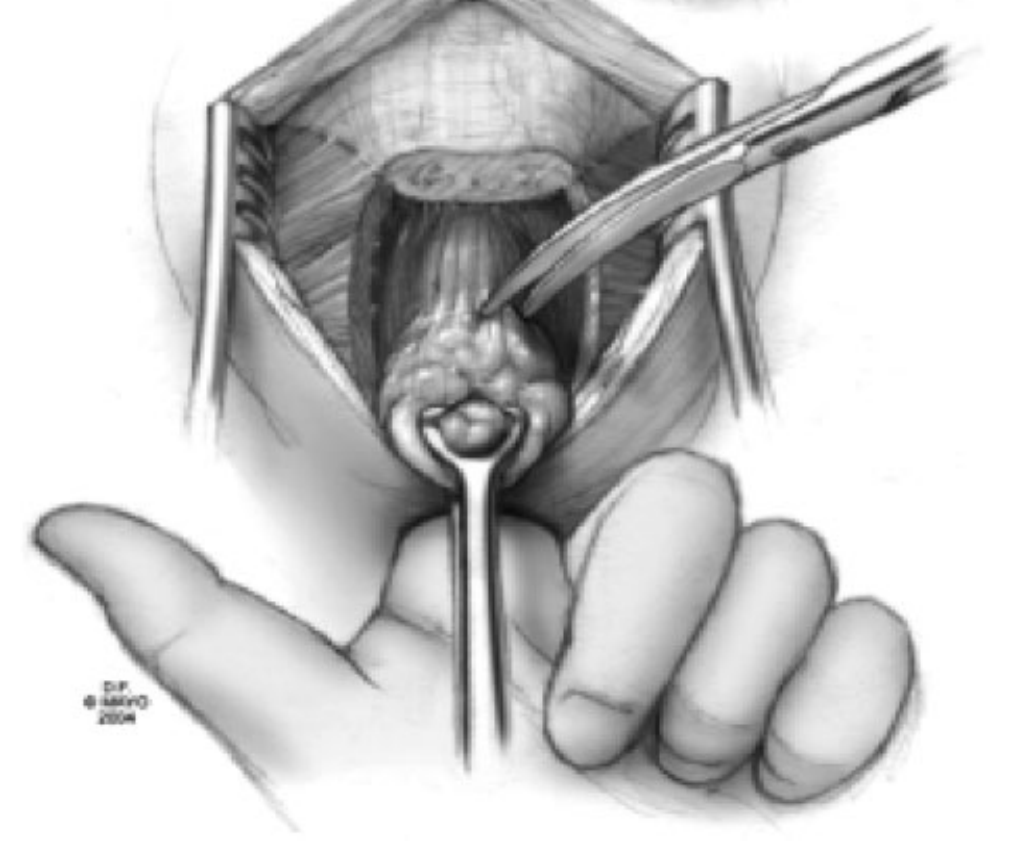

Figure 6 Posterior approach. (A) Positioning, (B) coccygectomy, and (C) the index finger applying pressure within the anal canal, exposing the lesion and facilitating dissection. From Dozois et al. ${ }^{8}$ Reprinted with permission of the American Society of Colon and Rectal Surgery. 
completeness of resection with incomplete resection being predisposed to higher recurrence rates. ${ }^{1}$ After a median follow-up of 22 months, Glasgow et $\mathrm{al}^{25}$ reported a $0 \%$ recurrence rate. Lev-Chelouche et $\mathrm{al}^{24}$ reported the complete excision of 21 benign tumors, with no local recurrence at 10-year follow-up. Finally, Buchs et $\mathrm{al}^{28}$ reported that of the 16 patients that underwent complete resection, one patient had local recurrence at 5-year follow-up.

Survival and recurrence rates after surgical resection for malignant lesions are based on tumor biology in conjunction with adequate resection margins, and whether the tumor has been violated during the resection. The risk of local recurrence after a poor oncologic resection approaches $70 \%$ with decreased long-term survival prospects. ${ }^{51}$ Kaiser et $\mathrm{el}^{52}$ discovered that if the tumor was violated perioperatively the local recurrence rate increased from $28 \%$ to $64 \%$. Glasgow et $\mathrm{al}^{25}$ demonstrated a disease recurrence of all seven patients who underwent an acceptable resection for malignant disease. The median survival for this patient group was 61 months. The literature on malignant retrorectal tumors reports a poor prognosis. Wang et $\mathrm{al}^{53}$ reported their series of 22 patients with malignant retrorectal tumors that included five chordomas and seven leiomyosarcomas. No preoperative biopsy was obtained and no neoadjuvant therapy was attempted. Postoperative chemotherapy and radiotherapy was used on selected patients. The 5-year survival rate was $41 \%$. Lev-Chelouche et $\mathrm{al}^{30}$ reported 21 cases of malignant retrorectal tumors that included nine chordomas. They achieved an $80 \%$ complete resection rate in 12 retrorectal malignancies, excluding chordomas. The local recurrence rate for these malignancies was $67 \%$, with a $50 \%$ survival rate. Chordomas in this series had a complete resection rate of $66 \%$, a local recurrence rate of $44 \%$, and a survival rate of $89 \%$. No preoperative biopsy was performed; the majority of recurrences occurred in patients with incomplete resection. Bergh et $\mathrm{al}^{22}$ observed a local recurrence rate of $44 \%$ and a 10 -year survival rate of $84 \%$ in their series on chordomas. Jao et $\mathrm{al}^{5}$ reported a 5 -year survival rate of $80 \%$, a 10 -year survival rate of $50 \%$ after resection, and a 5-year survival rate for malignant retrorectal tumors other than chordomas of $17 \%$. The authors attributed the low 5-year survival rate to poor quality of adjuvant therapy at that time. ${ }^{8}$ The literature from the past 50 years demonstrates that the 10 -year survival rate for the most common retrorectal tumor (chordoma) after surgical resection ranges from 15 to $84 \%$ at single institutions. ${ }^{5,22} \mathrm{McM}$ aster et $\mathrm{al}^{20}$ evaluated the 5- and 10-year survival rate of 400 chordomas at nine registries within the National Cancer Institute's Surveillance, Epidemiology and End Result program (NSEER) from 1973-1995. The 5-year and 10-year survival rates for sacral chordomas were $74 \%$ and $32 \%$, respectively. This study likely portrays the incidence and long-term survival of these lesions in the general population.

\section{CONCLUSION}

Tumors that arise in the retrorectal (presacral) space are uncommon lesions that present with nonspecific signs and symptoms, which lead to difficulty in diagnoses. A high clinical suspicion is required for diagnosis. Crosssectional imaging is mandatory for evaluation of retrorectal tumors, and it assists in planning the correct surgical approach by determining the extent of the tumor and nature of the lesion-solid or cystic. Preoperative tissue diagnosis with image-guided biopsy is essential for the management of solid and heterogeneously cystic tumors. Purely cystic lesions should not be biopsied unless there is a high suspicion of malignancy. Finally, these tumors must be managed with a multidisciplinary approach to maximize successful treatment in this $\mathrm{pa}^{-}$ tient population.

\section{REFERENCES}

1. Hobson KG, Ghaemmaghami V, Roe JP, Goodnight JE, Khatri VP. Tumors of the retrorectal space. Dis Colon Rectum 2005;48(10):1964-1974

2. Johnson WR. Postrectal neoplasms and cysts. Aust N Z J Surg 1980;50(2):163-166

3. Cody HS III, Marcove RC, Quan SH. Malignant retrorectal tumors: 28 years' experience at Memorial Sloan-Kettering Cancer Center. Dis Colon Rectum 1981; 24(7):501-506

4. Uhlig BE, Johnson RL. Presacral tumors and cysts in adults. Dis Colon Rectum 1975;18(7):581-589

5. Jao SW, Beart RW Jr, Spencer RJ, Reiman HM, Ilstrup DM. Retrorectal tumors. Mayo Clinic experience, 1960-1979. Dis Colon Rectum 1985;28(9):644-652

6. Gordon PH. Retrorectal tumors. In: Gordon PH, Nivatvongs S, eds. Principles and Practice of Surgery for the Colon, Rectum and Anus. St. Louis, MO: Quality Medical Publishing; 1999: 427-445

7. Young-Fadok TM, Dozois EJ. Retrorectal tumors. In: Yeo CJ, ed. Shackelford's Surgery of the Alimentary Tract. Philadelphia: Saunders; 2007:2299-2311

8. Dozois EJ, Jacofsky DJ, Dozois RR. Presacral tumors. In: Wolff BG, Fleshman JW, Beck DE, et al, eds. The ASCRS Textbook of Colon and Rectal Surgery. New York: Springer; 2007:501-514

9. Gunterberg B, Kewenter J, Petersén I, Stener B. Anorectal function after major resections of the sacrum with bilateral or unilateral sacrifice of sacral nerves. Br J Surg 1976;63(7): $546-554$

10. Stewart RJ, Humphreys WG, Parks TG. The presentation and management of presacral tumours. Br J Surg 1986;73(2): 153-155

11. Abel ME, Nelson R, Prasad ML, Pearl RK, Orsay CP, Abcarian H. Parasacrococcygeal approach for the resection of retrorectal developmental cysts. Dis Colon Rectum 1985; 28(11):855-858 
12. Hassan I, Wietfeldt ED. Presacral tumors: diagnosis and management. Clin Colon Rectal Surg 2009;22(2):84-93

13. Bullard Dunn K. Retrorectal tumors. Surg Clin North Am 2010;90(1):163-171

14. Williams B. Cerebrospinal fluid pressure changes in response to coughing. Brain 1976;99(2):331-346

15. Oren M, Lorber B, Lee SH, Truex RC Jr, Gennaro AR. Anterior sacral meningocele: report of five cases and review of the literature. Dis Colon Rectum 1977;20(6): 492-505

16. Izant RJ Jr, Filston HC. Sacrococcygeal teratomas. Analysis of forty-three cases. Am J Surg 1975;130(5):617-621

17. Waldhausen JA, Kolman JW, Vellios F, Battersby JS. Sacrococcygeal teratomas. Surgery 1963;54:933-949

18. Hickey RC, Martin RG. Sacrococcygeal teratomas. Ann N Y Acad Sci 1964;114:951-957

19. Fuchs B, Dickey ID, Yaszemski MJ, Inwards CY, Sim FH. Operative management of sacral chordoma. J Bone Joint Surg Am 2005;87(10):2211-2216

20. McMaster ML, Goldstein AM, Bromley CM, Ishibe N, Parry DM. Chordoma: incidence and survival patterns in the United States, 1973-1995. Cancer Causes Control 2001;12(1):1-11

21. Woodfield JC, Chalmers AG, Phillips N, Sagar PM. Algorithms for the surgical management of retrorectal tumours. Br J Surg 2008;95(2):214-221

22. Bergh P, Kindblom LG, Gunterberg B, Remotti F, Ryd W, Meis-Kindblom JM. Prognostic factors in chordoma of the sacrum and mobile spine: a study of 39 patients. Cancer 2000; 88(9):2122-2134

23. Miyahara M, Saito T, Nakashima K, et al. Sacral chordoma developing two years after low anterior resection for rectal cancer. Surg Today 1993;23(2):144-148

24. Lev-Chelouche D, Gutman M, Goldman G, et al. Presacral tumors: a practical classification and treatment of a unique and heterogeneous group of diseases. Surgery 2003;133(5): 473-478

25. Glasgow SC, Birnbaum EH, Lowney JK, et al. Retrorectal tumors: a diagnostic and therapeutic challenge. Dis Colon Rectum 2005;48(8):1581-1587

26. Singer MA, Cintron JR, Martz JE, Schoetz DJ, Abcarian H. Retrorectal cyst: a rare tumor frequently misdiagnosed. J Am Coll Surg 2003;196(6):880-886

27. Sobrado CW, Mester M, Simonsen OS, Justo CR, deAbreu JN, Habr-Gama A. Retrorectal tumors complicating pregnancy. Report of two cases. Dis Colon Rectum 1996;39(10): 1176-1179

28. Buchs N, Taylor S, Roche B. The posterior approach for low retrorectal tumors in adults. Int J Colorectal Dis 2007;22(4): 381-385

29. Zhang H, Yoshikawa K, Tamura K, et al. Carbon-11methionine positron emission tomography imaging of chordoma. Skeletal Radiol 2004;33(9):524-530

30. Luken MG III, Michelsen WJ, Whelan MA, Andrews DL. The diagnosis of sacral lesions. Surg Neurol 1981;15(5):377383

31. Böhm B, Milsom JW, Fazio VW, Lavery IC, Church JM, Oakley JR. Our approach to the management of congenital presacral tumors in adults. Int J Colorectal Dis 1993;8(3): 134-138

32. Eilbert FR. Expert commentary on retrorectal tumors: spectrum of disease, diagnosis and surgical management. Perp Col Rect Surg 1990;3:252-255
33. Pardes JG, Schneider M, Koizumi J, Engel IA, Auh YH, Rubenstein W. Percutaneous needle biopsy of deep pelvic masses: a posterior approach. Cardiovasc Intervent Radiol 1986;9(2):65-68

34. Triller J, Maddern G, Kraft P, Heidar A, Vock P. CT-guided biopsy of pelvic masses. Cardiovasc Intervent Radiol 1991; 14(1):63-68

35. Rapport RL II, Ferguson GS. Dorsal approach to presacral biopsy: technical case report. Neurosurgery 1997;40(5):10871088

36. Bodne DJ, Carrasco CH, Richli WR. Transosseous air contrast CT-guided needle biopsy of a cystic neoplasm. Cardiovasc Intervent Radiol 1993;16(2):122-123

37. Gupta S, Nguyen HL, Morello FA Jr, et al. Various approaches for CT-guided percutaneous biopsy of deep pelvic lesions: anatomic and technical considerations. Radiographics 2004;24(1):175-189

38. Yarram SG, Nghiem HV, Higgins E, Fox G, Nan B, Francis IR. Evaluation of imaging-guided core biopsy of pelvic masses. AJR Am J Roentgenol 2007;188(5):1208-1211

39. Syed R, Bishop JA, Ali SZ. Sacral and presacral lesions: cytopathologic analysis and clinical correlate. Diagn Cytopathol 2010; November 2 [Epub ahead of print]

40. Casali PG, Messina A, Stacchiotti S, et al. Imatinib mesylate in chordoma. Cancer 2004;101(9):2086-2097

41. Varga PP, Bors I, Lazary A. Sacral tumors and management. Orthop Clin North Am 2009;40(1):105-123, vii

42. Hof H, Welzel T, Debus J. Effectiveness of cetuximab/ gefitinib in the therapy of a sacral chordoma. Onkologie 2006; 29(12):572-574

43. Yang H, Zhu L, Ebraheim NA, et al. Surgical treatment of sacral chordomas combined with transcatheter arterial embolization. J Spinal Disord Tech 2010;23(1):47-52

44. Dozois EJ, Malireddy KK, Bower TC, Stanson AW, Sim FH. Management of a retrorectal lipomatous hemangiopericytoma by preoperative vascular embolization and a multidisciplinary surgical team: report of a case. Dis Colon Rectum 2009;52(5):1017-1020

45. MacCarty CS, Waugh JM, Coventry MB, Cope WF Jr. Surgical treatment of sacral and presacral tumors other than sacrococcygeal chordoma. J Neurosurg 1965;22(5): 458-464

46. Bax NM, van der Zee DC. The laparoscopic approach to sacrococcygeal teratomas. Surg Endosc 2004;18(1): $128-130$

47. Konstantinidis K, Theodoropoulos GE, Sambalis G, et al. Laparoscopic resection of presacral schwannomas. Surg Laparosc Endosc Percutan Tech 2005;15(5):302-304

48. Chen Y, Xu H, Li Y, et al. Laparoscopic resection of presacral teratomas. J Minim Invasive Gynecol 2008;15(5):649-651

49. Wolpert A, Beer-Gabel M, Lifschitz O, Zbar AP. The management of presacral masses in the adult. Tech Coloproctol 2002;6(1):43-49

50. Localio SA, Eng K, Ranson JHC. Abdominosacral approach for retrorectal tumors. Ann Surg 1980;191(5):555-560

51. Casali PG, Stacchiotti S, Sangalli C, Olmi P, Gronchi A. Chordoma. Curr Opin Oncol 2007;19(4):367-370

52. Kaiser TE, Pritchard DJ, Unni KK. Clinicopathologic study of sacrococcygeal chordoma. Cancer 1984;53(11):25742578

53. Wang JY, Hsu CH, Changchien CR, et al. Presacral tumor: a review of forty-five cases. Am Surg 1995;61(4):310-315 